\title{
A New Compliance Control Approach for Traveling-Wave Ultrasonic Motors
}

\author{
S. W. Chung and K. T. Chau, Senior Member, IEEE
}

\begin{abstract}
This paper presents a new approach for compliance control using a traveling-wave ultrasonic motor. Its objective is to demonstrate the effectiveness of using duty ratios and frequencies as control variables for both preload and stiffness control. At the home position, the motor is engaged in a standing-wave mode operation and provides self-locking torque. When the motor is deflected, it is engaged in a traveling-wave mode operation to provide a reacting torque. The torque is varied according to the deflection to provide the desired stiffness. Experiments have confirmed the effectiveness of this method. This approach can also be readily deployed into many force-feedback and haptic applications such as robotic locomotion.
\end{abstract}

Index Terms-Compliance, torque control, ultrasonic motor.

\section{NOMENCLATURE}

$V_{m} \quad$ Voltage amplitude of the driving voltage sources.

$\omega \quad$ Angular frequency of the driving voltage sources.

$f \quad$ Frequency of the driving voltage sources.

$\beta \quad$ Phase difference of the two driving voltage sources.

$L \quad$ Length of a single piezoelectric element.

$h \quad$ Thickness of a single piezoelectric element.

$q \quad$ Vertical downward force exerted from the pressure disc.

$T_{f} \quad$ Horizontal force exerted on the stator beam from the piezoelectric element.

$M \quad$ Moment generated by the piezoelectric element.

$F_{M} \quad$ Vertical upward force obtained from $M$ of phase $A$.

$w \quad$ Vertical deflection of the beam along the neutral plane.

$M_{q} \quad$ Bending moment from the vertical downward force $q$.

$x \quad$ Horizontal displacement along the beam.

$k \quad$ Angular displacement period of the square-wave moment $M$.

$\lambda \quad$ Displacement period of the square-wave moment $M$.

$q_{o} \quad$ Amplitude of the square-wave moment $M_{q}$.

$F_{M} \quad$ Vertical upward force obtained from $M$ of phase $A$.

$F_{o} \quad$ Amplitude of the force $F_{M}$.

$\alpha \quad$ Phase difference of the position of the infinite long beam where phase $A$ and phase $B$ apply.

$F_{M}^{\prime} \quad$ Vertical downward force obtained from $M$ of phase $B$.

Manuscript received January 26, 2005; revised May 29, 2007. This work was supported by a grant (Project HKU7159/03E) from the Research Grants Council, Hong Kong, China.

The authors are with the Department of Electrical and Electronic Engineering, The University of Hong Kong, Hong Kong (e-mail: ktchau@eee.hku.hk).

Color versions of one or more of the figures in this paper are available online at http://ieeexplore.ieee.org.

Digital Object Identifier 10.1109/TIE.2007.903936
$F_{R}$

$T$

$\Gamma_{S 1}$

$\Gamma_{S 2}$

$D_{f}$

$\Gamma_{S}$

$M_{\text {beam }}$

E

$J$

$S$

$\rho$

$A_{s}$

$c$

$F_{A}$

$M_{A}$

$w_{m}$

$F_{\text {Ao }}$

$w_{t}$

$w_{x}$

$\delta$

$Q$

$R$

L

C

$Q_{m}$

$\varphi$

$i_{m}$

$r$

$\Gamma_{\text {drive }}$

$\Gamma_{\text {load }}$

$\Gamma_{d}$

$\Gamma_{f}$

$\Gamma_{\text {ref }}$

$\theta$
Resultant of $F_{M}$ and $F_{M}^{\prime}$.

Period of the driving voltage sources.

Minimum self-locking torque.

Maximum self-locking torque.

Duty ratio of period the rotor experiences $\Gamma_{S 1}$ over the whole period $T$.

Average frictional torque.

Bending moment of the infinite long beam.

Young's modulus of the infinite long beam.

Second moment of area of the infinite long beam.

Shearing force from the resultant moment.

Mass per unit length of the infinite long beam.

Cross-sectional area of the infinite long beam.

Damping factor of the motor.

Tangential force due to the external load torque.

Moment obtained from $F_{A}$.

Maximum vertical deflection of the beam.

Amplitude of the tangential force $F_{A}$.

Vertical deflection function with time $t$ as the only independent variable.

Vertical deflection function with displacement $x$ as the only independent variable.

Proportional constant relating $4 M_{o} / \pi$ and $V_{m}$.

Equivalent charge representing the vertical deflection of the stator.

Equivalent resistance representing the damping factor of the motor.

Equivalent inductance representing the mass of the motor.

Equivalent capacitance representing the stiffness of the motor.

Equivalent charge amplitude of the deflection of the stator.

Phase lag of the deflection of the stator.

Equivalent current amplitude representing the rate of change of $Q_{m}$.

Effective mean radius of the TUSM.

Driving torque obtained from the piezoelectric effect of the TUSM.

Load torque applied to the TUSM.

Equivalent torque obtained from the downward force $q$.

Frictional torque between the rotor and the stator.

Reference self-locking torque.

Angular deflection of the motor shaft from the home position.

Stiffness specified for compliance control. 


\section{INTRODUCTION}

$\mathbf{T}$ HE traveling-wave ultrasonic motor (TUSM) drive is very suitable to servo applications, particularly in direct servo or gearless drive. It offers high torque at low speed, is lightweight, has a compact size, has a fast response, has no electromagnetic interference (EMI), and quietly operates. A TUSM requires two ac sinusoidal voltages with finite phase difference to be excited into rotation. Hence, its speed can be manipulated by varying the voltage amplitude, the frequency, and the phase difference of the two sources.

There have been researches in deploying this actuator in robotic manipulators and prosthetic arms. Both applications require position and speed control as well as compliance control. Numerous approaches have been explored to perform active position and speed control [1]-[9]. Various degrees of success have been achieved. Recently, these authors have also proposed a direct pulsewidth-modulation (PWM) approach, which combines frequency control and PWM over the external dc supply, to allow a more efficient and cost-effective method for active motion control using the TUSM drive [5].

On the other hand, comparatively few researches have been done on compliance control. The unique design of the TUSM provides a very large self-locking torque under static condition [10]. This is in contrast to a traditional electromagnetic motor, which provides minimum friction when no electricity is applied. This implies that the TUSM lacks compliance characteristics. It is particularly a challenge to vary this torque. Many have attempted to model the TUSM to relate the controllable inputs to the motor torque. An equivalent circuit approach has been presented as a useful analysis tool [10]; yet, it does not completely relate the parameters to physical properties. There are also various analytical models proposed [11]-[15]. In addition, the finite-element method has been employed to obtain a transient and steady-state response [16], [17]. As those models are too complex for control purposes, researchers have employed empirical methods to perform compliance control. Kato et al. [18] have utilized phase difference as a control variable for stiffness control. Ito et al. [19] have also demonstrated compliance control of an electromyography-controlled prosthetic forearm using an ultrasonic motor. Alternatively, Giraud et al. [20] has developed a model-based control for haptic stick application in avionics using frequency as the control variable. However, these approaches cannot specify the preload torque, in contrast to general spring-loaded application. The phase control used in this paper is also not commonly employed in embedded microcontroller application [5]. This increases hardware cost and complexity. Alternatively, Nishihori et al. [21] have proposed a PWM control. This approach continuously drives the TUSM in clockwise $(\mathrm{CW})$ and then counterclockwise (CCW) direction with a proper period. When driven at a high frequency, the motor can dramatically reduce its self-locking torque. By varying the duty ratio, the torque can be controlled. Unfortunately, engaging the TUSM in continuous motion produces rapid wear and quickly deteriorates its life expectancy. Kaneko et al. [22] have followed up this approach by inserting an OFF period between the $\mathrm{CW}$ and $\mathrm{CCW}$ periods. As a result, it improves the working life of the TUSM. However, it still engages the TUSM in continuous motion during operation. Thus, continuous wearing occurs whether there is rotation or not.

In this paper, a new approach is proposed and implemented for compliance control using the TUSM. Preload specification is achieved by relieving the self-locking torque of the TUSM. The motor is under a standing-wave mode operation at the home position. As a result, the rotor is changed between a "float" state and a "locked" state in a rapid successive fashion. The time lengths of the two states are determined by the amplitude of the standing wave. By varying the ratio between the time of the "float" state and that of the "locked" state, the selflocking torque can be controlled. The direct PWM method, which controls the duty ratios of the gate signals to the power electronics drive, is used to control the input voltage amplitudes. Since the TUSM produces no motion under the standingwave mode, no wearing occurs. On the other hand, to provide spring-like characteristics, the motor is under a traveling-wave mode when it is deflected from the home position. Thus, a reacting torque is generated. The spring-like characteristics are critical, particularly in robotic application. When it collides with a human, the human can experience an increasing effort that pushes him instead of a sudden impact, which can produce harm. Similarly, the direct PWM method is used to control the voltage amplitude, which governs the magnitude of the torque. By incorporating a position sensor, the torque can be varied according to the deflection. This allows stiffness, or the spring constant, be specified. The frequency of the voltage sources is also used as another control variable to increase the controllable span.

In Section II, the working principle of the TUSM will be briefly described. Both self-locking torque control and reacting torque control will be discussed. The system setup is illustrated in Section III. Section IV will show the torque characteristics. Section V will illustrate the control schemes. The experimental results are then presented and discussed in Section VI. Finally, conclusions will be drawn in Section VII.

\section{WORKING PRINCIPLE OF THE TUSM}

In contrast to the traditional electromagnetic motors that employ an electromagnetic effect, a TUSM utilizes a piezoelectric effect to convert electrical energy into mechanical motion. Hence, its components, as well as its operating principle, are fundamentally different from that of electromagnetic motors. Fig. 1 illustrates its basic mechanical components. The stator, which is designed with a comb-tooth surface, is attached below with sectors of piezoelectric elements. Those piezoelectric elements either contract or expand when they are subject to a voltage. They are divided into sectors instead of a complete ring so that they can be applied with different voltages. They also behave differently when the same voltage is applied. The rotor is pressed against the stator by means of a pressure spring disc, which is located directly above. It should be noted that the spring as shown is for illustration only. In an actual situation, it is not a normal spring but a pressure disc. A more detailed working principle and a component description can be found in [10]. 


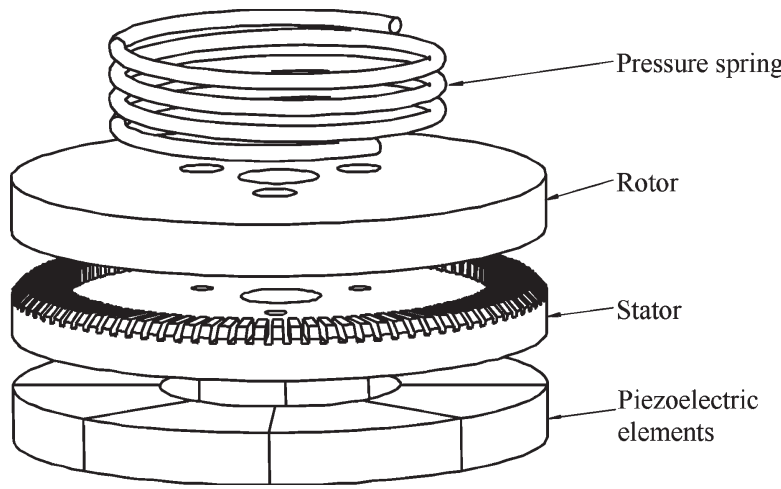

Fig. 1. Basic components of TUSM.

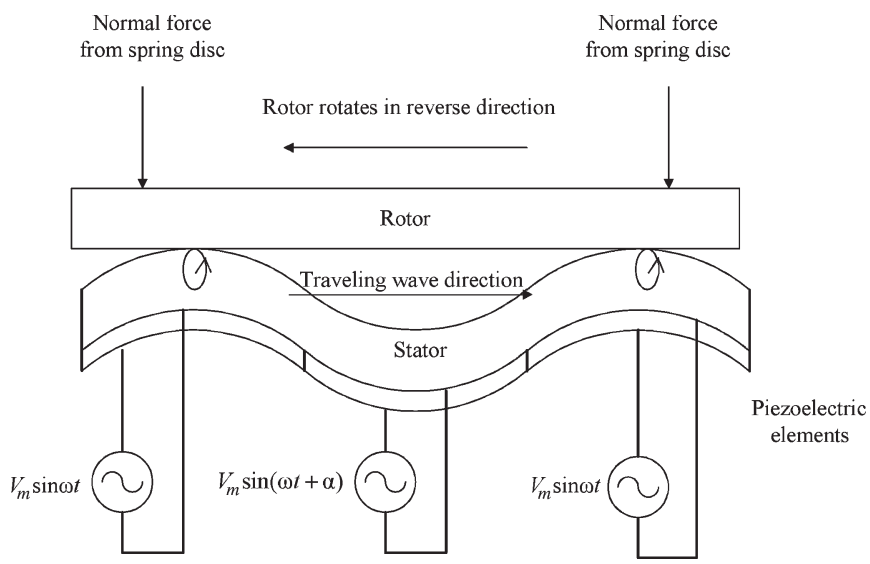

Fig. 2. Operating principle of TUSM.

Basically, the operating principle of the TUSM is due to the mechanical vibrations of piezoelectric elements, as a result of voltages applied on them. Hence, they produce a traveling wave in the stator. The surface interaction between the stator and the rotor creates a driving torque in the rotor. As shown in Fig. 2, piezoelectric elements are positioned at an appropriate distance from one another. One is fed by the phase A voltage source $V_{m} \sin \omega t$ to produce a mechanical vibration, whereas another is fed by the phase B voltage source $V_{m} \sin (\omega t+\beta)$ to generate another vibration, where $V_{m}$ is the amplitude, $\omega=$ $2 \pi f$ is the angular version of frequency $f$, and $\beta$ is the phase difference of the two driving voltage sources. Each voltage source generates a mode of vibration throughout the stator, and their superposition forms a traveling wave, which propagates along the stator. Depending on which phase of the voltage leads the other, the traveling wave will either travel in the righthand direction or in the left-hand direction. Elliptical motion can be observed at the wave crests of the surface of the stator. Such motion produces a tangential force at the contact surface between the stator and the rotor. Making use of a spring disc to keep a rigid rotor in pressure contact with the stator, the rotor can be driven to travel in the opposite direction of that of the traveling wave by this tangential force at the contact surface. Although the rotor moves only about $1 \mu \mathrm{m}$ in an individual cycle of the elliptical motion, this can add up to a speed of several centimeters per second at ultrasonic frequencies. The

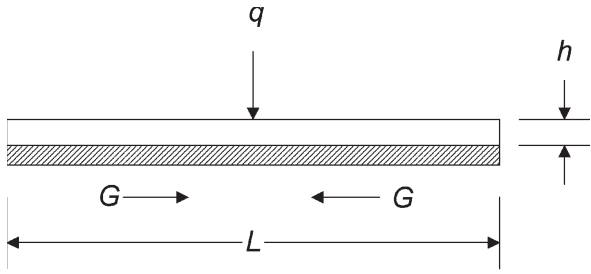

(a)

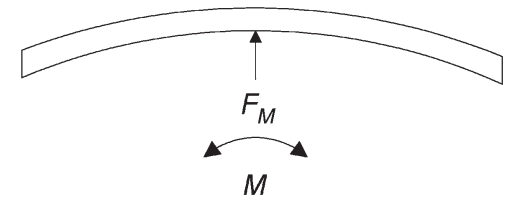

(b)

Fig. 3. Model of beam bending.

ring form structure provides a floating end condition, or acts as an infinite long beam for the mechanical vibration, which is essential for the generation of the traveling wave. In addition, without excitation by a proper electrical power source, the rotor cannot be rotated as it is pressed against the stator, hence producing a large self-locking torque.

\section{A. Self-Locking Torque Control}

The basic operation of the TUSM can be further illustrated by using the theory of beam bending. Fig. 3 shows a simple straight beam of length $L$ and thickness $h$. It represents the stator to which a piezoelectric element (the shaded portion) is attached. The beam is subject to a vertical downward force $q$ due to the pressure disc directly above the rotor. When a voltage is applied to the piezoelectric element, it contracts and thus results in a horizontal force $T_{f}$ that is exerted on the beam. As a result, a piezoelectric driving moment $M$ is formed, and, hence, a vertical upthrust $F_{M}$ is created, which are given by

$$
\begin{aligned}
M & =T_{f} h \\
F_{M} & =\frac{2 M}{L} .
\end{aligned}
$$

The magnitude of $F_{M}$ needs to be greater than $q$ to create beam bending. In such a situation, a vertical deflection $w$ results. The moment that is generated by the spring disc is $-q L / 2$. The negative sign indicates that the direction of the moment is opposite to that from the piezoelectric element.

To simplify the discussion, an infinite long beam, which is excited by a single phase of a sinusoidal voltage with piezoelectric elements attached below is considered. From Fig. 4, it can be seen that a square wave of moment is applied to the beam. Both the piezoelectric driving moment $M$, owing to the application of sinusoidal voltage $A$, and the moment $M_{q}$, owing to the vertical spring force, are square-wave 


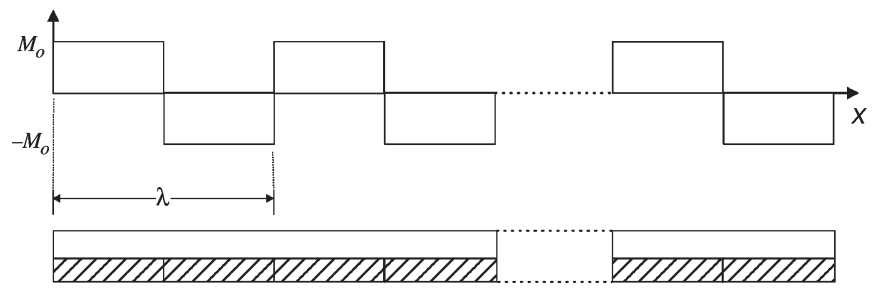

Fig. 4. Piezoelectric driving moment applied along the infinite long beam.

moments. This can be represented by using the Fourier series as

$$
\begin{aligned}
M= & \left(\frac{4 M_{o}}{\pi} \sum_{n=1}^{\infty} \frac{1}{2 n-1} \sin (2 n-1) \frac{2 \pi}{\lambda} x\right) \sin \omega t \\
M_{q}= & -\frac{L}{2}\left(\frac{16 q_{o}}{\pi^{2}} \sum_{n=1}^{\infty} \frac{1}{2 n-1} \sin (2 n-1) \frac{2 \pi}{\lambda} x\right) \\
& \times\left(\sum_{n=1}^{\infty} \frac{1}{2 n-1} \sin \frac{(2 n-1) \pi}{2} \sin (2 n-1) \omega t\right)
\end{aligned}
$$

where $x$ is the horizontal displacement along the beam, $\lambda$ is the displacement period of the square-wave moment with an amplitude $M_{o}, q_{o}$ is the magnitude of the downward force from the spring disc applying to each element, $f$ is the frequency, and $\omega$ is the angular frequency of the voltage source. The negative sign of $M_{q}$ indicates that the direction of the moment is opposite to $M$. Both $\omega$ and $M_{o}$ are governed by the applied voltage of the two-phase inverter. Since the stator of the TUSM is usually of comb-teeth design to purposely amplify the fundamental component of oscillation, $M$ and $M_{q}$ can be approximated as

$$
\begin{aligned}
M & =\frac{4 M_{o}}{\pi} \sin k x \sin \omega t \\
M_{q} & =-\frac{8 q_{o} L}{\pi^{2}} \sin k x \sin \omega t
\end{aligned}
$$

where $k=2 \pi / \lambda$. It should also be noted that when $M$ goes negative, i.e., the piezoelectric elements expand, the vertical force turns downward. Suppose the stator rests on an immovable plane. The downward force is transmitted back to push up the rotor at the two ends. This is due to the reaction from the plane. The vertical force $F_{M}$ can be written as

$$
\begin{aligned}
F_{M} & =\left(\frac{2}{L} \frac{4 M_{o}}{\pi} \sin k x\right) \sin \omega t \\
& =F_{o} \sin k x \sin \omega t
\end{aligned}
$$

where $F_{o}=8 M_{o} / \pi L$. From (7), it can be seen that a standing wave of force is generated along the beam.

When the long beam is excited by another phase of sinusoidal voltage $B$ at the same time, another driving moment results. Another standing wave of vertical force is generated. The resultant force can be obtained by superimposing the two standing waves. Suppose that the second sinusoidal voltage has the same voltage amplitude but is lagged by a phase of $\beta$. At the same time, the voltage applies to the long beam at a position that is different from the previous by $\alpha$. Then, the force of the second phase $F_{M}^{\prime}$ can be expressed as

$$
F_{M}^{\prime}=F_{o} \sin (k x+\alpha) \sin (\omega t+\beta)
$$

The resultant vertical force $F_{R}$ can be found by

$$
\begin{aligned}
F_{R} & =F_{M}+F_{M}^{\prime} \\
& =F_{o}[\sin k x \sin \omega t+\sin (k x+\alpha) \sin (\omega t+\beta)] .
\end{aligned}
$$

From (9), two special scenarios can be considered. The first one is when $\alpha=\beta=\pi / 2$. Then, (9) can be rewritten as

$$
\begin{aligned}
F_{R} & =F_{o}\left[\sin k x \sin \omega t+\sin \left(k x+\frac{\pi}{2}\right) \sin \left(\omega t+\frac{\pi}{2}\right)\right] \\
& =F_{o}[\sin k x \sin \omega t+\cos k x \cos \omega t] \\
& =F_{o} \cos (k x-\omega t) .
\end{aligned}
$$

Since $F_{R}$ describes a traveling wave, there always exists a sinusoidal force wave along the beam. That means that once the forces that are generated from bending overcome the downward force $q$ from the pressure disc, at some positions along the beam, there always exist forces that can overcome $q$. Furthermore, as previously discussed, when $F_{R}$ goes negative, the force is transmitted back to positive. That means that the rotor is constantly being pushed up by the bending of the stator or is essentially "floating" over the stator. The friction between them becomes minimum. At the same time, the surface of the stator also generates a traveling wave that drives the rotor into rotation. The second one is when $\alpha=\pi / 2$, and $\beta=0$. Then, (9) can be rewritten as

$$
\begin{aligned}
F_{R} & =F_{o}\left[\sin k x \sin \omega t+\sin \left(k x+\frac{\pi}{2}\right) \sin \omega t\right] \\
& =F_{o}[\sin k x \sin \omega t+\cos k x \sin \omega t] \\
& =\sqrt{2} F_{o} \sin \omega t \sin \left(k x+\frac{\pi}{4}\right) .
\end{aligned}
$$

Instead of a traveling wave, a standing wave is generated. First, there is no self-generated motion. When there is no external torque, no wearing occurs between the surfaces. Second, $F_{R}$ is sometimes greater, and sometimes smaller, than $q$. Suppose that the dynamic of the stator is neglected. It can be argued that when $F_{R}>q$, the rotor is "floating" over the stator. When $F_{R}<q$, the rotor is "locking" to the stator, as bending is not allowed. The situation is illustrated further in Fig. 5. In a period $T$, the rotor is "floated" when $t_{0}<t<t_{1}$. It experiences a minimum self-locking torque $\Gamma_{S 1}$. In the other time, it is "locked" and experiences the maximum self-locking torque $\Gamma_{S 2}$. Thus, a duty ratio $D_{f}$ is established as

$$
D_{f}=\frac{t_{1}-t_{0}}{T} .
$$

Incidentally, since the TUSM operates at an ultrasonic frequency around $40 \mathrm{kHz}$, a weighted averaging effect 


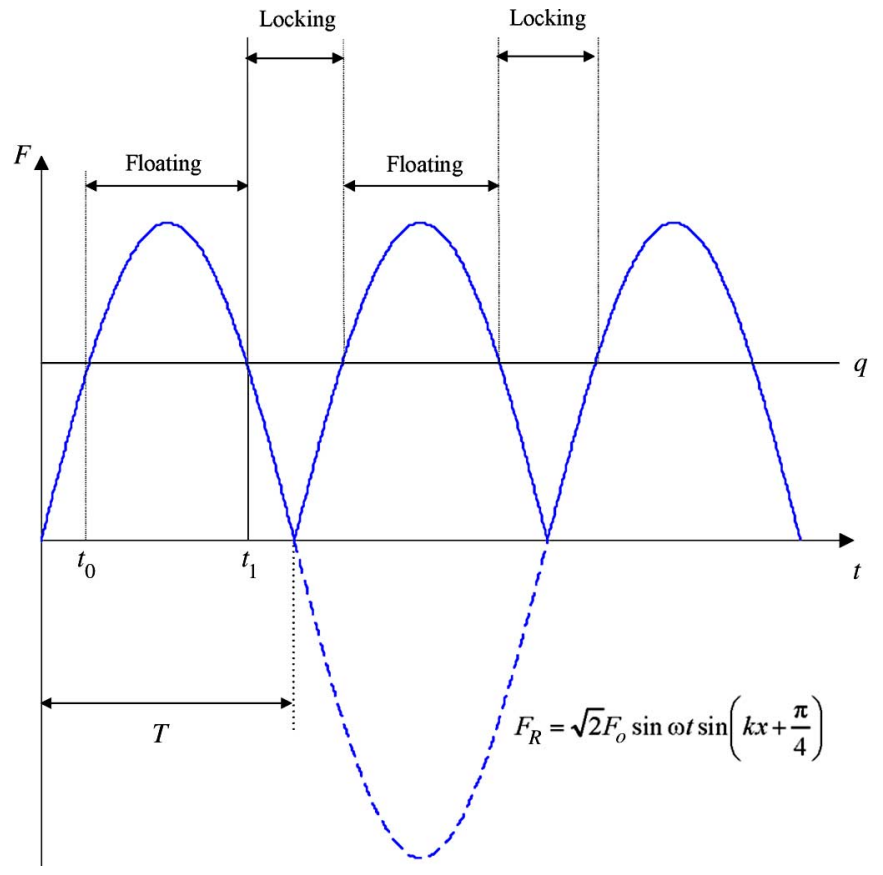

Fig. 5. Relationship of $F_{R}$ and $q$.

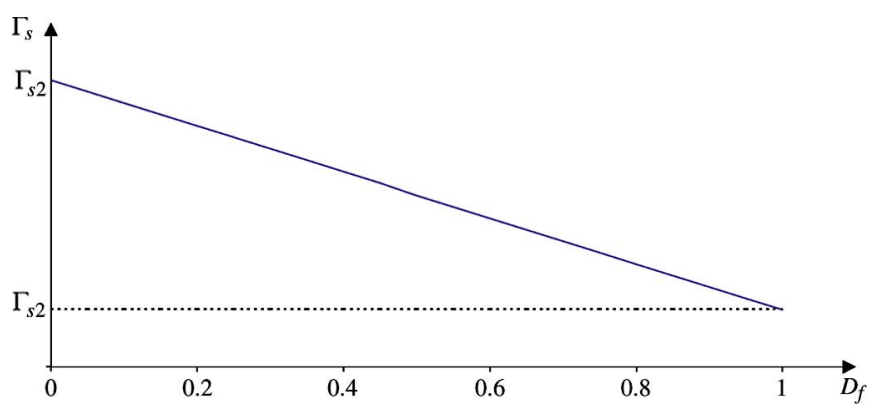

Fig. 6. Relationship of $\Gamma_{S}$ and $D_{f}$.

of the two frictional torques can be expected. Hence, the frictional torque $\Gamma_{S}$ that the rotor-stator surface produced will be

$\Gamma_{S}=\left\{\begin{array}{ll}\Gamma_{S 2}\left(1-D_{f}\right)+\Gamma_{S 1} D_{f} & \text { when } 0 \leq D_{f}<1 \\ \Gamma_{S 1} & \text { when } D_{f}=1\end{array}\right.$.

Fig. 6 shows the relationship between $\Gamma_{S}$ and $D_{f}$. From (13), it is obvious that the self-locking torque is determined by $D_{f}$, which increases as the force amplitude $F_{o}$ increases. This can be observed readily in Fig. 5 . At the same time, $F_{o}$ is governed by $M_{o}$, as shown in (7). On the other hand, $M_{o}$ is controlled by $V_{m}$, which is the voltage amplitude of the two voltage sources that are applied over the piezoelectric elements. As a result, the self-locking torque can be controlled by varying the voltage amplitude of the two sinusoidal voltage sources $A$ and $B$.

However, the above discussion implicitly assumes that the stator and the piezoelectric elements are "rigid body." Since the TUSM operates at resonance, the dynamic of the stator has to be accounted. The inertia, the damping characteristics, and the elasticity of the stator and the piezoelectric elements all have an impact on the self-locking torque during the proposed operation. As revealed in the authors' previous paper [8], according to the simple beam bending theory

$$
\begin{aligned}
M_{\text {beam }} & =-E J \frac{\partial^{2} w}{\partial x^{2}} \\
S & =\frac{\partial}{\partial x}\left(M+M_{\text {beam }}+M_{q}\right)
\end{aligned}
$$

where $M_{\text {beam }}$ is the bending moment along the beam, $E$ is the Young's modulus of the beam, $J$ is the second moment of area of the beam, and $w$ is the vertical deflection along the neutral plane of the beam. $S$ is the shearing force that is produced as a result of the moment. By Newton's second law of motion, it yields

$$
\rho A_{s} \frac{\partial^{2} w}{\partial t^{2}}+c \frac{\partial w}{\partial t}=\frac{\partial S}{\partial x}
$$

where $\rho$ is the mass per unit length, $A_{s}$ is the cross-sectional area of the beam, and $c$ is the damping factor. Hence, combining (6), (14)-(16), it gives

$$
\begin{aligned}
\rho A_{s} \frac{\partial^{2} w}{\partial t^{2}}+c \frac{\partial w}{\partial t}+ & E J \frac{\partial^{4} w}{\partial x^{4}} \\
& =-\left(\frac{4 M_{o}}{\pi}-\frac{8 q_{o} L}{\pi^{2}}\right) \sin k x \sin \omega t
\end{aligned}
$$

As discussed previously, "floating" of rotor results when $F_{R}$ is greater than $q$, or the term $\left(4 M_{o} / \pi\right)-\left(8 q_{o} L / \pi^{2}\right)$ is greater than zero. In such situations, bending motion occurs. That means $w$ becomes nonzero. Thus, the criteria for "floating" of rotor can be further interpreted as $w>0$ for $t_{0}<t<t_{1}$ and "locking" when $w=0$.

Equation (17) gives a more complete description than Fig. 5 by taking the dynamics of the stator and the piezoelectric elements into account. It can readily be found that the inertial force of the stator $\rho A_{s}\left(\partial^{2} w / \partial t^{2}\right)$, the damping force $c(\partial w / \partial t)$, and the elastic force $E J\left(\partial^{4} w / \partial x^{4}\right)$ also affect the duration when $w>0$. One immediate observation is that even when $\left(4 M_{o} / \pi\right)-\left(8 q_{o} L / \pi 2\right)$ turns negative, or when $F_{R}$ is smaller than $q$, the rotor will be reverted to a "locked" state. $D_{f}$, thus, is expected to be longer than that in Fig. 5. In fact, as $D_{f}$ becomes larger, the continuous vibration of the beam itself can lead the rotor into a continuous "floated" state, as shown later in Section IV.

\section{B. Reacting Torque Control}

To provide a spring back torque, the TUSM is engaged in the traveling-wave mode operation when it is deflected from the home position. The model can be illustrated in Fig. 7. It is similar to Fig. 3 except that a tangential force $F_{A}$, which represents the force due to the external torque, is added. Similar to $q$, it produces a moment $M_{A}$, which should be in the opposite direction to $M . M_{A}$ can be expressed as

$$
M_{A}=-F_{A}\left(w_{m}+h\right)
$$




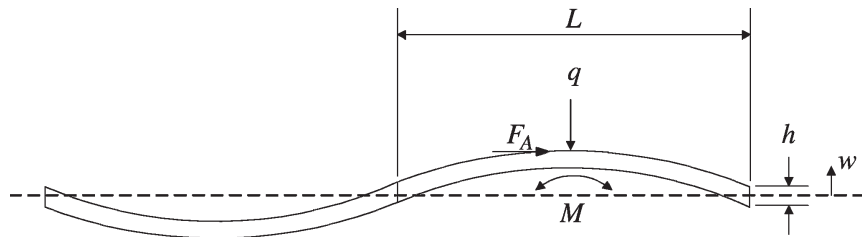

Fig. 7. Reacting torque model.

where $w_{m}$ is the maximum vertical deflection throughout the beam. Since $h>>w_{m}$, (18) can be approximated as

$$
M_{A} \approx-F_{A} h .
$$

When the infinite long beam model is considered, it yields

$$
\begin{aligned}
M_{A}= & -\frac{16}{\pi^{2}} F_{A o} h\left(\sum_{n=1}^{\infty} \frac{1}{2 n-1} \sin (2 n-1) k x\right) \\
& \times\left(\sum_{n=1}^{\infty} \frac{1}{2 n-1} \sin (2 n-1) n \omega t\right) \\
\approx & -\frac{16}{\pi^{2}} F_{A o} h \sin k x \sin \omega t
\end{aligned}
$$

where $F_{A o}$ is the external force amplitude. Hence, under the reacting torque control, (15) and (17) become

$$
\begin{aligned}
& S=\frac{\partial}{\partial x}\left(M+M_{\text {beam }}+M_{q}+M_{A}\right) \\
& \rho A_{s} \frac{\partial^{2} w}{\partial t^{2}}+c \frac{\partial w}{\partial t}+E J \frac{\partial^{4} w}{\partial x^{4}} \\
&=-\left(\frac{4 M_{o}}{\pi}-\frac{8 q_{o} L}{\pi^{2}}-\frac{16 F_{A o} h}{\pi^{2}}\right) \sin k x \sin \omega t .
\end{aligned}
$$

As $\sin \omega t=-\cos ((\pi / 2)+\omega t)$, the dummy variable is rewritten as $\omega t=(\pi / 2)+\omega t$. Hence, it gives

$$
\begin{aligned}
\rho A_{s} \frac{\partial^{2} w}{\partial t^{2}} & +c \frac{\partial w}{\partial t}+E J \frac{\partial^{4} w}{\partial x^{4}} \\
& =\left(\frac{4 M_{o}}{\pi}-\frac{8 q_{o} L}{\pi^{2}}-\frac{16 F_{A o} h}{\pi^{2}}\right) \sin k x \cos \omega t .
\end{aligned}
$$

By using the method of separating variable

$$
w=w_{t}(t) w_{x}(x)=w_{t} \sin k x .
$$

it can be further expressed as

$$
\begin{aligned}
\frac{\rho A_{s}}{k^{2}} \frac{d^{2} w_{t}}{d t^{2}}+\frac{c}{k^{2}} & \frac{d w_{t}}{d t}+E J k^{2} w_{t} \\
& =\left(\frac{4 M_{o}}{\pi}-\frac{8 q_{o} L}{\pi^{2}}-\frac{16 F_{A o} h}{\pi^{2}}\right) \cos \omega t .
\end{aligned}
$$

Since the piezoelectric effect implies that $V_{m} \propto M_{o}$, it gives $4 M_{o} / \pi=\delta V_{m}$, where $\delta$ is a constant. Hence, (25) is rewritten as

$$
\begin{aligned}
\frac{\rho A_{s}}{\delta k^{2}} \frac{d^{2} w_{t}}{d t^{2}}+\frac{c}{\delta k^{2}} & \frac{d w_{t}}{d t}+\frac{E J k^{2}}{\delta} w_{t} \\
& =\left[V_{m}-\frac{8}{\delta \pi^{2}}\left(q_{o} L+2 F_{A o} h\right)\right] \cos \omega t .
\end{aligned}
$$

Taking $Q=w_{t} / \delta, L=\rho A / k^{2}, R=c / k^{2}$, and $1 / C=$ $E J k^{2},(26)$ can be used to establish an equivalent circuit model as given by

$$
\begin{aligned}
L \frac{d^{2} Q}{d t^{2}}+R \frac{d Q}{d t} & +\frac{1}{\mathrm{C}} Q \\
& =\left[V_{m}-\frac{8}{\delta \pi^{2}}\left(q_{o} L+2 F_{A o} h\right)\right] \cos \omega t
\end{aligned}
$$

Since the natural response is too fast for mechanical motion, it is ignored. To find the particular solution $Q=Q_{m} \sin (\omega t-$ $\varphi$ ), we need to first derive $d Q / d t$ and $d^{2} Q / d t^{2}$ and then substitute them into (27). Hence, it yields

$$
\begin{aligned}
& \left(\omega^{2} Q_{m} L \sin \varphi+\omega Q_{m} R \cos \varphi-\frac{Q_{m}}{C} \cos \varphi\right) \cos \omega t \\
& +\left(-\omega^{2} Q_{m} L \cos \varphi+\omega Q_{m} R \sin \varphi+\frac{Q_{m}}{C} \cos \varphi\right) \sin \omega t \\
& \quad=\left[V_{m}-\frac{8}{\delta \pi^{2}}\left(q_{o} L+2 F_{A o} h\right)\right] \cos \omega t
\end{aligned}
$$

By comparing both sides of (28), it gives

$$
-\omega^{2} Q_{m} L \cos \varphi+\omega Q_{m} R \sin \varphi+\frac{Q_{m}}{C} \cos \varphi=0 .
$$

Since $Q_{m} \neq 0$, it results in

$$
\begin{aligned}
-\omega^{2} L \cos \varphi+\omega R \sin \varphi+\frac{1}{C} \cos \varphi & =0 \\
\tan \varphi & =\frac{\omega L-\frac{1}{\omega C}}{R}
\end{aligned}
$$

It can be seen that $\varphi$ is determined by the parameters of the TUSM only and is independent of the loading application.

From (28), the comparison also gives

$$
\begin{aligned}
& \omega^{2} Q_{m} L \sin \varphi+\omega Q_{m} R \cos \varphi-\frac{Q_{m}}{C} \cos \varphi \\
&=V_{m}-\frac{8}{\delta \pi^{2}}\left(q_{o} L+2 F_{A o} h\right) .
\end{aligned}
$$

Taking $i_{m}=\omega Q_{m}$, which is actually the phase current amplitude of the TUSM, and using the relationship that $\omega^{2} L \sin \varphi+$ $\omega R \cos \varphi-(1 / C) \cos \varphi=\omega \sqrt{\left(\omega L-(1 / \omega C)^{2}+R^{2}\right.}$, (31) can be further expressed as

$$
F_{A o}+\frac{q_{o} L}{2 h}=\frac{\delta \pi^{2}}{16 h}\left(V_{m}-i_{m} \sqrt{\left(\omega L-\frac{1}{\omega C}\right)^{2}+R^{2}}\right) .
$$

When $r$ is the effective mean radius of the TUSM, the load torque is given by $\Gamma_{\text {load }}=F_{A o} r$. Also, taking $\Gamma_{d}=q_{o} L r / 2 h$, 
(32) becomes

$$
\begin{aligned}
\Gamma_{\text {load }}+\Gamma_{d} & =A_{V \Gamma}\left(V_{m}-i_{m} \sqrt{\left(\omega L-\frac{1}{\omega C}\right)^{2}+R^{2}}\right) \\
& =\Gamma_{\text {drive }}
\end{aligned}
$$

where $A_{V \Gamma}=\delta \pi^{2} r / 16 h$, and $\Gamma_{\text {drive }}$ is actually the driving torque obtained from the piezoelectric effect of the TUSM. $\Gamma_{d}$ can be considered as the equivalent torque from the downward force $q$ that is produced by the pressure disc.

Therefore, (33) demonstrates that voltage amplitude can be employed to control $\Gamma_{\text {drive }}$, although it does not linearly increase with increasing $V_{m}$. When $V_{m}$ increases, $i_{m}$ will also increase. As a result, as shown in (28), there will only be a marginal increase in $\Gamma_{\text {drive }}$.

Rewriting (33) leads to $\Gamma_{\text {load }}=\Gamma_{\text {drive }}-\Gamma_{d}$. It implies that the driving torque needs to overcome the bending from the vertical downward force $q$ before it can contribute any torque for the load. In case of compliance application under the travelingwave mode operation, $\Gamma_{\text {load }}>\Gamma_{\text {drive }}-\Gamma_{d}$. However, in such a situation, a slip occurs. An additional frictional torque $\Gamma_{f}$ exists because of the surface interaction between the rotor and the stator. It should be noted that when $\Gamma_{\text {load }}>\Gamma_{\text {drive }}-\Gamma_{d}+\Gamma_{f}$, the motor system complies with external torque, and motion occurs. Increasing $\Gamma_{\text {drive }}$ by increasing $V_{m}$ will reduce the difference. When $\Gamma_{\text {load }}=\Gamma_{\text {drive }}-\Gamma_{d}+\Gamma_{f}$, the motor system reaches equilibrium, and motion stops. When $\Gamma_{\text {drive }}$ is increased further, but $\Gamma_{\text {load }}>\Gamma_{\text {drive }}-\Gamma_{d}$, the motor system will still be in equilibrium. The reason is that $\Gamma_{f}$ will start decreasing due to its nature. Only when $\Gamma_{\text {drive }}-\Gamma_{d}>\Gamma_{\text {load }}$ does the motor system start rotation in the reverse direction. Hence, because of $\Gamma_{f}$, a hysteresis exists in employing the TUSM for a spring-like stiffness control system.

It can be seen that both the self-locking torque and the reacting torque can be controlled by $V_{m}$ under the standing-wave and traveling-wave mode operations, respectively. Furthermore, voltage control is also a common technique that is employed in power electronics and microcontroller technologies. Hence, it is chosen as the control variable for compliance control using the TUSM.

\section{System SETUP}

A DSP microcontroller TMS320F240 is selected as the core of hardware implementation. It is capable of generating a highfrequency signal as well as quickly performing a complex computation. Fig. 8 shows the system control hardware. Initially, the control software is programmed by using the $\mathrm{C}$ language. Then, the compiled program is downloaded into the TMS320F240. After downloading, it can run in the standalone mode. This TMS320F240 is externally driven by a $20-\mathrm{MHz}$ crystal, which is capable of generating high-frequency PWM signals with variable phase differences, variable frequencies, and variable duty ratios. These digital PWM signals are directly fed to the two-phase inverter circuit, hence increasing the electrical noise immunity and the control reliability. Also, its built-in edgesignal detection feature allows it to directly gather data from

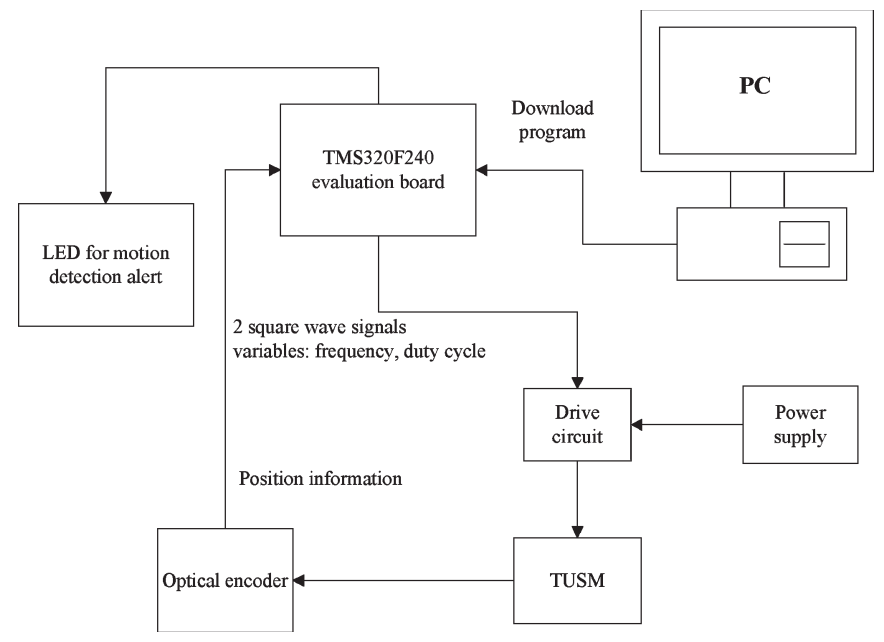

Fig. 8. Hardware implementation block diagram.

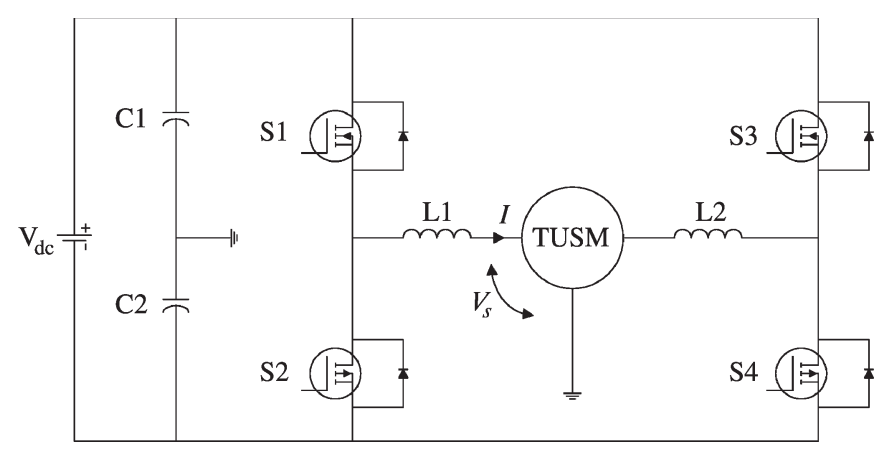

Fig. 9. Two-phase inverter circuit for TUSM.

the optical encoder without signal conditioning. Furthermore, by adding a simple phase split circuit, one TMS320F240 can actually simultaneously control two TUSMs, which leads to favoring its application to mechatronics and robotics.

Fig. 9 shows the two-phase inverter circuit for the TUSM. It adopts an H-bridge configuration to provide the PWM switching voltages. Since the input terminals of the TUSM are inherently capacitive, two small inductors L1 and L2, of value $1 \mathrm{mH}$, are purposely added in series to its terminals so that resonance is obtained. The corresponding resonance can offer an advantageous feature of soft switching, hence reducing the switching loss and the EMI. Those power devices that connect to the positive rail are p-channel IRF9640 MOSFET, and those that connect to the negative rail are n-channel IRF740 MOSFET. Such an arrangement can minimize the required number of isolated dc supplies for MOSFET gate driving.

The TUSM is coupled to a dc servomotor, which serves as an external torque generator. The TUSM used is a USR60 from Shinsei Industries Co. Ltd. The specification is listed in Table I. To find the motor torque value, current is supplied to the dc servomotor through a dc supply. As a result, the dc servomotor generates torque to try to rotate the TUSM. When the current is increased so that the torque from the dc servomotor overcomes the self-locking torque of the TUSM, motion results. The motion is detected by an optical encoder, which feeds back signal to the DSP microcontroller. The torque 
TABLE I

TUSM SPECIFICATION

\begin{tabular}{ll}
\hline Rated speed $(\mathrm{rpm})$ & 90 \\
Rated torque $(\mathrm{Nm})$ & 0.31 \\
Rated phase voltage $(\mathrm{V})$ & 100 \\
Rated driving frequency $(\mathrm{kHz})$ & 40.5 \\
\hline
\end{tabular}

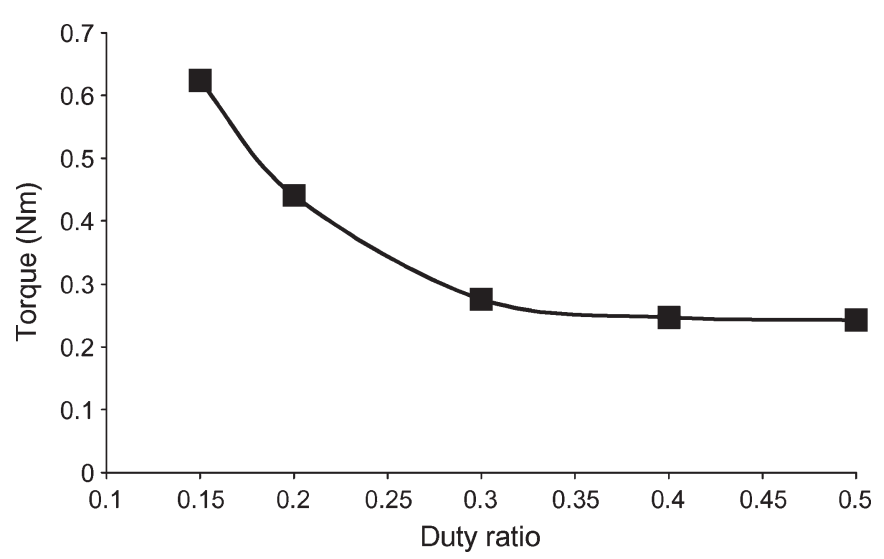

Fig. 10. Self-locking torque characteristics versus duty ratio.

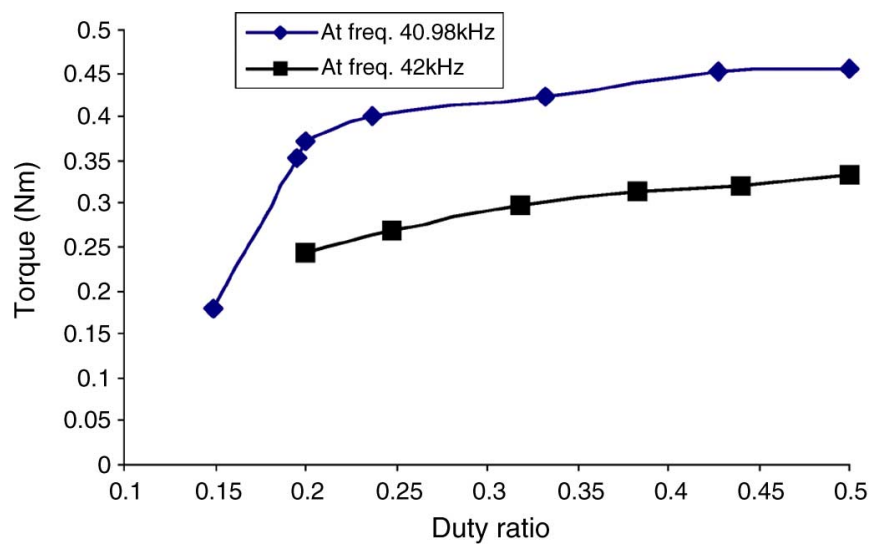

Fig. 11. Reacting torque characteristics versus duty ratio.

can then be found by multiplying the dc current with the torque constant of the de servomotor.

\section{TUSM TORQUE CHARACTERISTICS}

Fig. 10 shows the self-locking torque at different duty ratios of the PWM signals at $42 \mathrm{kHz}$ to the MOSFETs. The supply dc voltage is $120 \mathrm{~V}$. There is a reduction of the self-locking torque value with an increasing duty ratio. It rapidly drops at the initial stage and gradually levels off when the duty ratio reaches maximum. As discussed in Section II, because of the dynamics of the stator, the rotor eventually floats over the stator throughout the time when the phase voltage reaches a certain level. At this point, the self-locking torque is reduced to its minimum.

In contrast, the reacting torque increases with an increasing duty ratio, as shown in Fig. 11. However, the relationship is nonlinear. The duty ratio allows only a diminishing increase in the torque. On the other hand, different torque characteristics can be obtained at different driving frequencies. At a frequency of $40.98 \mathrm{kHz}$, the duty ratio control provides a greater range of the torque value. It should also be noted that the reacting torque

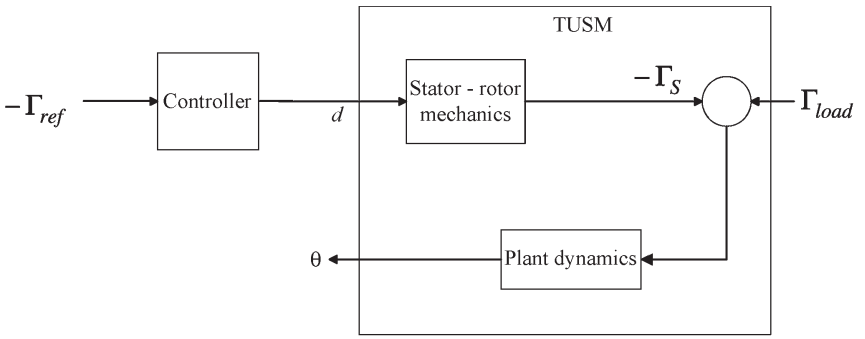

(a)

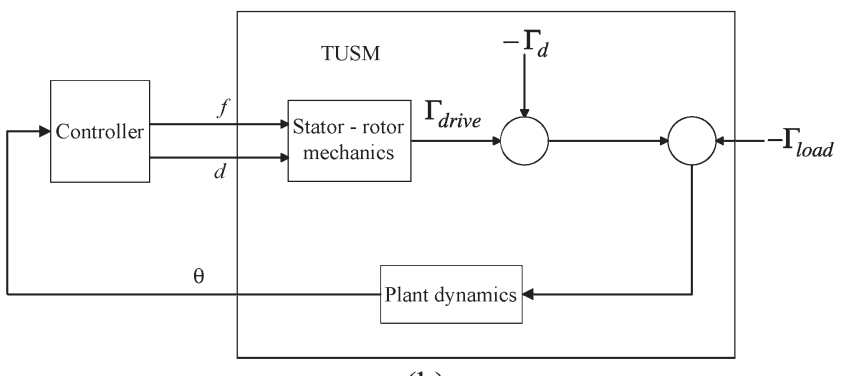

(b)

Fig. 12. Control synoptic. (a) Self-locking torque control. (b) Reacting torque control.

is produced after the duty ratio exceeds a certain threshold. This is because a threshold force from the piezoelectric effect is needed to overcome the vertical downward force $q$ from the pressure disc. Then, the torque sharply increases when the duty ratio increases from a low level. When the duty ratio is greater than 0.2 , the rate of increases significantly reduces. At a frequency of $42 \mathrm{kHz}$, the duty ratio provides a smaller range, but allows for a higher control resolution.

\section{Control Scheme}

To realize voltage amplitude control for the self-locking torque control and the reacting torque control, the duty ratio of the PWM signals to the gates of all the MOSFETs is controlled. It is known that the voltage amplitudes of the two resonant sinusoidal voltage waveforms proportionally respond to the duty ratio [8]. As a result, the self-locking torque and the reacting torque of the TUSM will respond as discussed in Section III. In addition, to expand the controllable torque span, frequency is also adopted as another controllable variable. Although phase control has the advantage of covering the whole controllable span [18] and shows a negligible dead zone at certain frequency and voltage [20], it does not offer the selflocking torque control. As a result, the preload torque cannot be specified. In addition, phase control is not commonly employed in general power electronics and microcontroller technologies. Hence, additional hardware is needed for implementation. This increases bulk and cost.

The control synoptic is illustrated in Fig. 12. The self-locking torque control is shown in Fig. 12(a). At this mode, the motor is engaged under standing-wave mode control. The sinusoidal voltages are at $42 \mathrm{kHz}$ and are in phase with each other. $-\Gamma_{\text {ref }}$ is the reference self-locking torque. The negative sign indicates that the torque opposes the externally applied torque. The controller then finds the corresponding duty ratio by means of table lookup according to Fig. 10. When the external load 


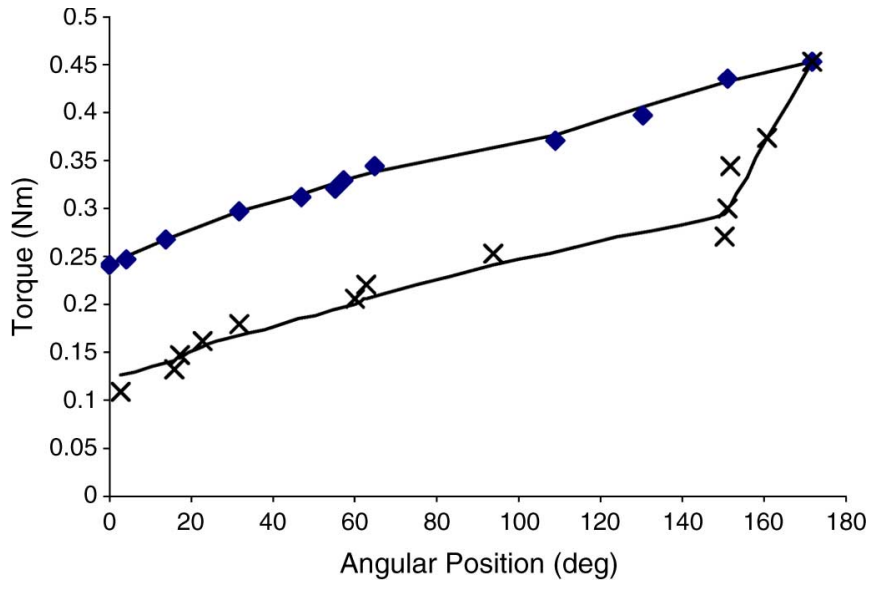

Fig. 13. Torque versus angular position with stiffness $0.00117 \mathrm{~N} \mathrm{~m} /{ }^{\circ}$ and preload $0.243 \mathrm{~N} \mathrm{~m}$.

torque is greater than $\Gamma_{\text {ref }}$, the optical encoder records a deflection $\theta$ of the motor shaft, and the system instantaneously switches to the reacting torque control, which is shown in Fig. 12(b). The motor is switched to traveling-wave mode control. The two voltages have a phase difference of $\pi / 2$. The controller first determines $\Gamma_{\text {ref }}$ according to $\theta$ and the designed stiffness $\kappa$. They are related by

$$
\Gamma_{\text {ref }}=\kappa \theta .
$$

The controller then determines the frequency by finding out that the range of $\Gamma_{\text {ref }}$ falls within, according to Fig. 11. The duty ratio is then obtained by table lookup using Fig. 11 again.

\section{RESUlTS}

Fig. 13 shows the torque against angular position. The preload torque is specified as $0.243 \mathrm{~N} \mathrm{~m}$. Stiffness is $0.00117 \mathrm{~N} \mathrm{~m} /{ }^{\circ}$. It can be seen that when the external torque is above the preload, the system is deflected from its home position. The system then generates an increasing reacting torque with increasing deflection. When the external torque is removed, it automatically returns to its home position. Also, hysteresis can be observed with gradual decreasing of external torque, as discussed in the previous section. Fig. 14 shows the corresponding frequency and duty ratio responses. It can be observed that to increase the reacting torque, the duty ratio is increased by the controller, as discussed in Sections IV and V. When the required torque exceeds the range controllable by the employed frequency, the controller responds by reducing the frequency, according to the characteristics obtained in Section V.

To demonstrate possible manipulation of preload and stiffness, another experimental result is obtained. Fig. 15 shows the result. The preload is raised to $0.271 \mathrm{~N} \mathrm{~m}$, and stiffness was $0.00104 \mathrm{~N} \mathrm{~m} /{ }^{\circ}$. Fig. 16 shows the corresponding frequency and duty ratio response. It can be noted that to provide a large self-locking torque, the duty ratio at home position is reduced, compared with that in Fig. 14. This agrees well with the discussion in Section IV and the characteristics shown in Section V. A constant self-locking torque for $3^{\circ}$ is designed to

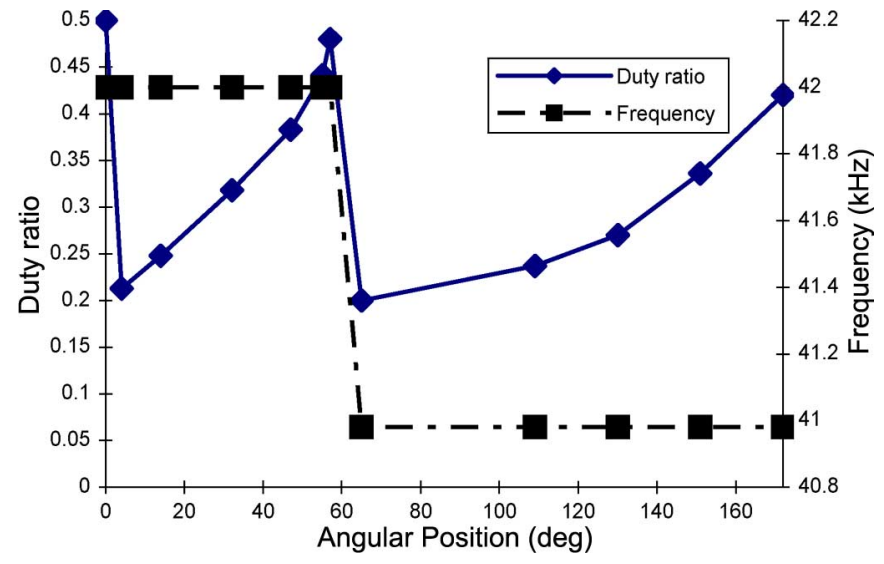

Fig. 14. Frequency and duty ratio versus angular position with stiffness $0.00117 \mathrm{~N} \mathrm{~m} /{ }^{\circ}$ and preload $0.243 \mathrm{~N} \mathrm{~m}$.

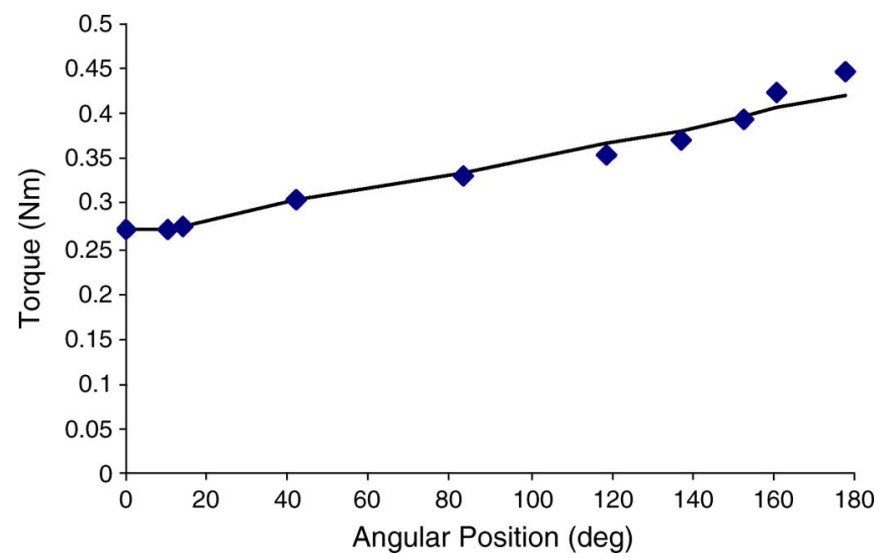

Fig. 15. Torque versus angular position with stiffness $0.00104 \mathrm{~N} \mathrm{~m} /{ }^{\circ}$ and preload $0.271 \mathrm{~N} \mathrm{~m}$.

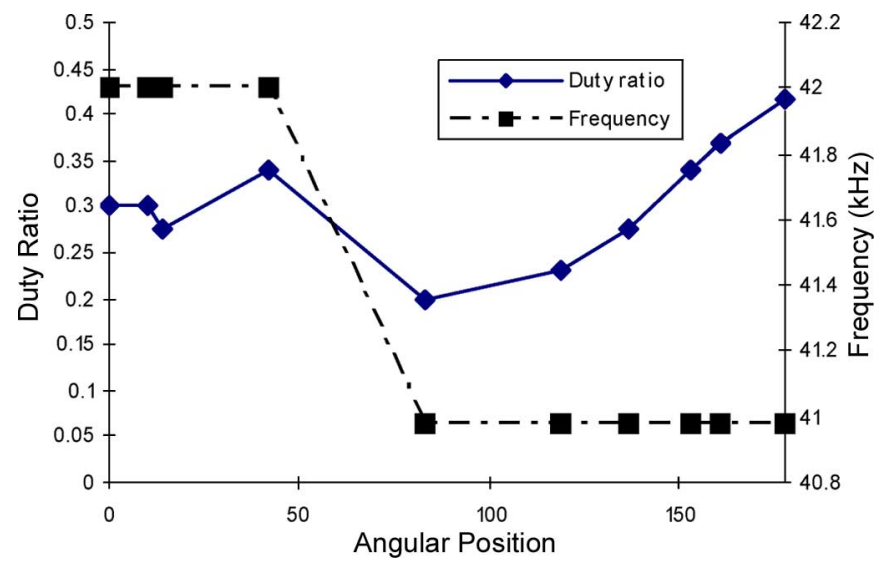

Fig. 16. Frequency and duty ratio versus angular position with stiffness $0.00104 \mathrm{~N} \mathrm{~m} /{ }^{\circ}$ and preload $0.271 \mathrm{~N} \mathrm{~m}$.

show the versatility of such a system. It can be clearly shown in Fig. 16. The duty ratio and the frequency are intentionally kept constant from home position to $3^{\circ}$ of deflection. The sudden drop of the duty ratio from 0.3 to 0.27 is a result of changing the standing-wave mode operation into the traveling-wave mode operation. A different duty ratio value is then needed to provide the reacting torque after the mode change. 


\section{CONCLUSION}

This paper has proposed a new method to achieve compliance control using the TUSM. The combination of the standingwave mode and traveling-wave mode operations allows both preload specification and stiffness control. By means of the duty ratio and the frequency control, the self-locking torque as well as the reacting torque can be controlled according to the angular position. The principle of control behind the system is discussed. An experiment also has demonstrated the feasibility of the proposed method.

\section{REFERENCES}

[1] F. J. Lin, "Fuzzy adaptive model-following position control for ultrasonic motor," IEEE Trans. Power Electron., vol. 12, no. 2, pp. 261-268, Mar. 1997.

[2] F. J. Lin, R. J. Wai, and R. Y. Duan, "Fuzzy neural networks for identification and control of ultrasonic motor drive with LLCC resonant technique," IEEE Trans. Ind. Electron., vol. 46, no. 5, pp. 999-1011, Oct. 1999.

[3] T. Senjyu, T. Kashiwagi, and K. Uezato, "Position control of ultrasonic motors using MRAC with dead-zone compensation," IEEE Trans. Ind. Electron., vol. 48, no. 6, pp. 1278-1285, Dec. 2001.

[4] K. T. Chau and S. W. Chung, "Servo position control of ultrasonic motors using fuzzy neural network," Electr. Mach. Power Syst., vol. 29, no. 3, pp. 229-246, 2001.

[5] K. T. Chau and S. W. Chung, "Servo speed control of traveling-wave ultrasonic motors using pulse width modulation," Electr. Power Compon. Syst., vol. 29, no. 8, pp. 707-722, 2001.

[6] Y. F. Peng, R. J. Wai, and C.-M. Lin, "Implementation of LLCC-resonant driving circuit and adaptive CMAC neural network control for linear piezoelectric ceramic motor," IEEE Trans. Ind. Electron., vol. 51, no. 1, pp. 35-48, Feb. 2004.

[7] N. Bonnail, D. Tonneau, F. Jandard, G.-A. Capolino, and H. Dallaporta, "Variable structure control of a piezoelectric actuator for a scanning tunneling microscope," IEEE Trans. Ind. Electron., vol. 51, no. 2, pp. 354-363, Apr. 2004.

[8] R.-J. Wai and C.-H. Tu, "Adaptive grey control for hybrid resonant driving linear piezoelectric ceramic motor," IEEE Trans. Ind. Electron., vol. 53, no. 2, pp. 640-656, Apr. 2006.

[9] R.-J. Wai and K.-H. Su, "Supervisory control for linear piezoelectric ceramic motor drive using genetic algorithm," IEEE Trans. Ind. Electron., vol. 53, no. 2, pp. 657-673, Apr. 2006.

[10] T. Sashida and T. Kenjo, An Introduction to Ultrasonic Motors. Oxford, U.K.: Clarendon, 1993.

[11] N. Hagood and A. McFarland, "Modeling of piezoelectric rotary ultrasonic motor," IEEE Trans. Ultrason., Ferroelectr., Freq. Control, vol. 42, no. 2, pp. 210-224, Mar. 1995.

[12] P. Hagedorn and J. Wallaschek, "Traveling wave ultrasonic motors. Part I: Working principle and mathematical modeling of the stator," J. Sound Vibration, vol. 144, no. 1, pp. 31-36, 1992.

[13] J. Mass and H. Grotstollen, "Averaged model of inverter-fed ultrasonic motors," in Proc. IEEE Power Electron. Spec. Conf., 1997, pp. 740-746.

[14] R. Fung, C. Yao, and D. Chang, "Dynamics and contact analysis of a bimodal ultrasonic motor," IEEE Trans. Ultrason., Ferroelectr., Freq. Control, vol. 46, no. 1, pp. 47-60, Jan. 1999.
[15] M. S. Tsai, C. H. Lee, and S. H. Hwang, "Dynamic modeling and analysis of a bimodal ultrasonic motor," IEEE Trans. Ultrason., Ferroelectr, Freq. Control, vol. 50, no. 3, pp. 245-256, Mar. 2003.

[16] Y. Kagawa, T. Tsuchiya, T. Yamabushi, and T. Furukawa, "Finite element simulation of dynamic responses of piezoelectric actuators," J. Sound Vibration, vol. 191, no. 4, pp. 519-538, 1996.

[17] T. Maeno and A. Miyaka, "Finite element analysis of the rotor/stator contact in a ring type ultrasonic motor," IEEE Trans. Ultrason., Ferroelectr. Freq. Control, vol. 39, no. 6, pp. 668-674, Nov. 1992.

[18] A. Kato, K. Ito, and M. Ito, "Compliance control of circular traveling wave motor," in Proc. Int. Conf. Ind. Electron., Control and Instrum., 1991, vol. 1, pp. 538-542.

[19] K. Ito, M. Pecson, Z. W. Luo, M. Yamakita, A. Kato, T. Aoya, and M. Ito, "Compliance control of an EMG-controlled prosthetic forearm using ultrasonic motors," in Proc. Int. Conf. Intell. Robots and Syst., 1994, vol. 3, pp. 1816-1823.

[20] F. Giraud, B. Semail, and J. T. Audren, "Analysis and phase control of a piezoelectric traveling-wave ultrasonic motor for haptic stick application," IEEE Trans. Ind. Appl., vol. 40, no. 6, pp. 1541-1549, Nov./Dec. 2004.

[21] K. Nishihori, S. Ohkuma, Y. Eryu, and T. Sugimoto, "Velocity control of ultrasonic motors for robot arms by pulse width modulation," in Proc. $3 \mathrm{rd}$ IEEE Int. Workshop Adv. Motion Control, 1994, pp. 823-829.

[22] M. Kaneko, T. Nishihara, and T. Tsuji, "Active control of self-locking characteristic of ultrasonic motor," in Proc. IEEE Int. Conf. Robot. Autom., 1995, vol. 3, pp. 2928-2934.

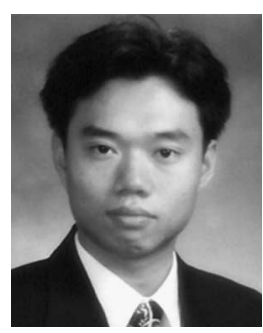

S. W. Chung was born in 1975 . He received the B.Eng. (Industrial Automation) degree in mechanical engineering and the M.Phil. degree in electrical and electronic engineering from The University of Hong Kong, Hong Kong, where he is currently working toward the Ph.D. degree in electrical and electronic engineering, in 1997 and in 2001, respectively.

His research interests are servomotor drives and control theory application.

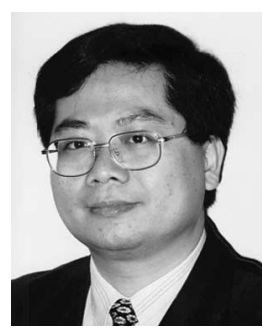

K. T. Chau (M'89-SM'04) received the first-class honors B.Sc.(Eng.), M.Phil., and Ph.D. degrees in electrical and electronic engineering from The University of Hong Kong, Hong Kong, in 1988, 1991, and 1993, respectively.

He is currently a Professor with the Department of Electrical and Electronic Engineering and the Director with the International Research Center for Electric Vehicles, both at The University of Hong Kong. His teaching and research interests focus on three main areas-electric vehicles, electric drives, and power electronics. In these areas, he has published over 200 refereed technical papers. He is also the coauthor of a monograph Modern Electric Vehicle Technology (London, U.K.: Oxford Univ. Press, 2001).

Dr. Chau was the recipient of the Outstanding Young Researcher Award in 2003, the University Teaching Fellowship Award in 2004, and the Award for Innovative Excellence in Teaching, Learning, and Technology in 2005. 